\title{
Treatment Supporters and Their Impact on Treatment Outcomes In Routine Tuberculosis Program Conditions In District Rawalpindi, Pakistan
}

\author{
MUNAWAR HUSSAIN SOOMRO, ${ }^{1,4}$ EJAZ QADEER, ${ }^{2}$ MUHAMMAD AMIR KHAN, ${ }^{3}$ ODD MORKVE ${ }^{4}$
}

\begin{abstract}
Background: Tuberculosis (TB) is a major cause of mortality and is affecting millions of people in third world countries. In DOTS patients are monitored facility based and treatment supporter based; by these two ways patients are observed for the treatment. The aim of the study was to explore the role of treatment supporters and their impact on the treatment outcomes.

Material and Method: The study was a cross-sectional survey within routine TB control program operational context. All sputum smear positive TB patients diagnosed, registered in public sector, urban and rural diagnostic centre during year 2008 with available outcomes were included in the study. Data was collected during AugustOctober 2010 from 15 health facilities of 45 I patients.

Results: The majority of the patients (89.6\%) were provided with treatment supporters. In 404 (89.6\%) cases in which treatment supporters were provided, 203 (50.2\%) were lady health worker, 46 (I I.4\%) were community health worker and health facility worker, and 155 (38.4\%) were family member and community volunteer. 384 (85. I\%) were categorized as "treatment success", 31 (6.9\%), as "transferred out", I 7 (3.8\%), as “dead”, I 6 (3.5\%) as "defaulted" and three (0.7\%) as "treatment failure". The treatment success rates in patients supervised by lady health worker, community health worker and health facility worker, and family member and community volunteer was $93.1 \%$, $89.1 \%$ and $73.5 \%$, respectively.
\end{abstract}

Conclusions: We found a significantly higher treatment success (93.1\%) in patients supervised by lady health workers compared to other types of treatment support. The overall treatment success rate was $85.1 \%$.

Keywords: Cross-sectional study, Outcomes; Pakistan; Treatment supporter; Tuberculosis.

\section{Introduction}

Tuberculosis (TB) is a contagious, airborne disease and remains a major public health problem globally. TB is a major cause of mortality and is affecting millions of people in low income and middle income countries. Worldwide one person out of three is infected with the mycobacterium tuberculosis (MTB). Each year about 9.4 million people develop TB disease globally and 1.7 million die of this disease. ${ }^{1}$

TB is mostly affecting developing countries and it has been reported that more than $85 \%$ of TB cases have been noticed in the developing countries. According to the World Health Organization (WHO), more than $80 \%$ of the new TB cases have been notified in 22 high burden countries, with Asia $55 \%$ and Africa $30 \%$ of cases. ${ }^{1}$ Along with the new cases, a

1. Department of Community Medicine, Shaheed Mohtarma Benazir Bhutto Medical University, Larkana, Pakistan.

2. National TB Control Program, Pakistan.

3. Association for Social Development, Pakistan.

4. Centre for International Health, University of Bergen, Norway. Correspondence : Dr. Munawar Hussain Soomro, Department of Community Medicine, Shaheed Mohtarma Benazir Bhutto Medical University, Larkana, Pakistan., Email address: munawar_soomro@hotmail.com significant number of re-treatment cases are being noticed. The factors associated with the resurgence of TB are low economic conditions (malnutrition, poverty, poor sanitation and homelessness), overcrowding, stress, alcohol, acquired immunodeficiency syndrome (AIDS), diabetes mellitus, immune deficiency states, corticosteroid therapy. ${ }^{2}$

Pakistan ranks number eight of the high burden countries. Directly observed treatment short-course (DOTS) is an internationally recommended treatment strategy for TB patients, and for their continuous follow-up during the treatment (3). DOTS rely on self referral of symptomatic individuals to health facilities, so called "passive case finding". WHO has set the millennium development goals (MDGs) to control TB with $70 \%$ case detection rate and $85 \%$ cure rate in the DOTS program. The national TB control program of Pakistan achieved countrywide DOTS coverage in 2005 .

There are many tasks involved in providing treatment support to the patients that are mainly carried out by the treatment supporter. ${ }^{4}$ A suitable and acceptable treatment supporter for the patient is the key to success for DOT and is mandatory at least for smear-positive patients in intensive 
phase and for the whole duration of treatment for re-treatment cases. The available treatment supporter options include: health facility based worker (HFW) i.e. health staff member working at the selected treatment centre; community health worker (CHW) i.e. any person formally associated with the health services and living close to the patient's place; community volunteer (CVT) like a suitable person selected from the community e.g. teachers, religious leaders, neighbours, co-workers and friends etc; lady health worker (LHW) i.e. a woman formally working with the national program for primary health care and family planning (PHC \& $\mathrm{FP})^{5,6}$. A family member (FM) or any person who is willing and acceptable to the patient and answerable to the health services can also be a treatment supporter. ${ }^{5}$

Objectives:

To explore the role of treatment supporters and their impact on the treatment outcomes in district and sub-district hospitals and rural health centers in Rawalpindi district Pakistan.

\section{Methodology}

It was a cross-sectional survey conducted during August to October 2010 within routine TB control program operational context. A quantitative research method was used to achieve the objectives of the study. The data were collected from 15 health facilities one district hospital, 4 sub-district hospitals, and 10 Rural Health Centers covering 98 Basic Health Units in the district Rawalpindi. A total of 451 sputum smear positive TB patients diagnosed and treated in public sector, urban and rural diagnostic centers during the year 2008 were registered.

Data was extracted from the patients TB treatment cards (TB01) and TB register (TB03) on a pre-designed form.

Inclusion criteria:

All smear positive TB patients registered during year 2008 in public sector district, sub-district hospitals and rural health centers in DOTS program with available treatment outcomes.

\section{Exclusion criteria:}

1. All smear negative pulmonary, extra-pulmonary cases other than sputum smear positive cases. 2. All private hospitals.

Data were recorded in a pre-designed form and were entered and analyzed by SPSS/PASWstats version 17. Descriptive statistics were applied, using frequencies and cross tabulation, and Pearson Chi-square test $\left(\chi^{2}\right)$ was used to compare group differences for categorical variables. The level of statistical significance was set at $\mathrm{P}<0.05$.

\section{Results}

Baseline characteristics:

Table 1 shows the distribution of the TB patients according to geography, gender and age. Fifty three percent of the patients were male. $53.4 \%$ of the registered patients lived in urban settings, whereas $46.6 \%$ of the patients were registered in rural settings.

For the data analysis purpose, patients were divided into three age groups ${ }^{7}$ :

Group 1: Age $<14$ yrs (Childhood)

Group 2: Age 15-54 yrs (Productive age)

Group 3: Age $>55$ yrs (Older age)

There were $14(3.1 \%)$ patients in the childhood group, 324 $(71.8 \%)$ in the productive age group and $113(25.1 \%)$ in the older age group.

\section{Treatment Support:}

The majority of the patients $(89.6 \%)$ were provided with treatment supporters, which were recorded on the TB01 form. In $404(89.6 \%)$ cases in which treatment supporters were provided, 203 (50.2\%) were LHW, 46 (11.4\%) were CHW/ HFW, and 155 (38.4\%) were FM/CVT.

Twenty four patients $(5.3 \%)$ had self-administered treatment (self-support) and in 23 patients (5.1\%) treatment support was not documented. The patients in the groups "self support" and "undocumented" were combined to "no treatment support".

$53.4 \%$ treatment supporters lived in urban settings and $46.6 \%$ in rural areas. Among treatment supporters $129(63.5 \%) \mathrm{LHW}$ belongs to rural setting and $112(72.3 \%) \mathrm{FM} / \mathrm{CVT}$ lives in urban setting. Among the $\mathrm{CHW} / \mathrm{HFW}$ most of the patients $25(54.3 \%)$ also lived in the rural setting. Among the patients with "no treatment support" $34(72.3 \%)$ lived in the urban setting. When comparing the group of patients with "treatment support" by LHW with patients "no treatment support", we found highly significant difference between urban and rural locations $\left(\chi^{2}=20.03, p=0.0000076\right)$. Similarly when compared the group of patients with "treatment support" by CHW/HFW with patients "no treatment support", we found a significant difference between urban and rural locations $\left(\chi^{2}=6.852, \mathrm{p}=0.00885\right)$ (Table-II).

In all registered patients, 237 (52.5\%) treatment supporters were male, while 214 (47.5\%) were female. Among all the registered patients 203 (45\%) were allocated to LHW, 102 males and 101 females. In FM/CVT male to female distribution was 81 (52.3\%), and 74 (47.7\%) respectively (Table-III). 
Table-IV shows that from all the registered patients, 324 (71.8\%) belonged to the productive age group (15-54 yrs). Most of them were provided with LHW 137 (67.5\%), CHW/ HFW 34 (73.9\%) and FM/CVT 118 (76.1\%) among treatment support, while $35(74.5 \%)$ with "no treatment support" also belonged to this age group. Among the older age group (e" $55 \mathrm{yrs}), 60$ (29.6\%) were provided with LHW, 10 (21.7\%) with CHW/HFW and 31 (20\%) were provided with FM/CVT type of treatment supporter, while $12(25.5 \%)$ were in the "no treatment support" group. There were only 14 (3.1\%) cases in the childhood age group ( $<14 \mathrm{yrs}$ ), and all were provide with treatment supporter.

Treatment outcomes:

Three hundred and eighty four (85.1\%) were categorized as "treatment success", 31 (6.9\%), as "transferred out", 17 (3.8\%), as "dead", $16(3.5 \%)$ as "defaulted" and $3(0.7 \%)$ as "treatment failure".

The majority of the registered patients with treatment success (189) were under the LHW as "treatment support" with urban to rural distribution of $72(35.5 \%)$ and $117(57.6 \%)$, respectively. One hundred and fourteen patients had FM/ CVT as treatment supporters with urban to rural distribution of $75(48.4 \%)$ and 39 (25.1\%), respectively. Similarly among the patients with "no treatment support" 40 were declared as treatment success, with urban to rural distribution of 30 $(63.8 \%)$ and $10(21.3 \%)$, respectively. When comparing the group of patients with "treatment support" by LHW with group "no treatment support", we found highly significant difference between urban and rural locations in treatment success $\left(\chi^{2}=18.2, P=0.00002\right)$. Similarly, when compared the group of patients with "treatment support" by $\mathrm{CHW} /$ HFW with the group "no treatment support", we found a significant difference between urban and rural locations in treatment success $\left(\chi^{2}=6.958, \mathrm{P}=0.008\right)$. The treatment success rate in LHW, CHW/HFW, and FM/CVT was 93.1\%, $89.1 \%$ and $73.5 \%$, respectively (Table-IV).
Table-I

Baseline characteristics of TB patient's geographic locations.

\begin{tabular}{lcc}
\hline Characteristics & \multicolumn{2}{c}{ Geography } \\
\cline { 2 - 3 } & $\begin{array}{c}\text { Urban } \mathrm{n}(\%) \\
241(53.4)\end{array}$ & $\begin{array}{c}\text { Rural } \mathrm{n}(\%) \\
210(46.6)\end{array}$ \\
\hline Gender & & \\
Male & $128(53.1)$ & $109(51.9)$ \\
Female & $113(46.9)$ & $101(48.1)$ \\
Age group & & \\
$<14$ yrs & $6(2.5)$ & $8(3.8)$ \\
$15-54$ yrs & $185(76.7)$ & $139(66.2)$ \\
$>55$ yrs & $50(20.8)$ & $63(30)$ \\
\hline
\end{tabular}

The majority of the registered patients with treatment success (189) were under the LHW as "treatment support" with male to female distribution of $91(44.8 \%)$ and $98(48.3 \%)$, respectively. One hundred and fourteen patients had FM/ CVT as treatment supporters with male to female distribution of $61(39.3 \%)$ and $53(34.2 \%)$, respectively. Similarly among the patients with "no treatment support" 40 were declared as treatment success, with male to female distribution of 23 (48.9\%) and $17(36.2 \%)$, respectively (Table-VI).

Most of the patients with "treatment support" from LHW were categorized as treatment success and were distributed in the three age groups as $6(3 \%), 130(64 \%)$ and $53(26.1 \%)$, respectively. One hundred and fourteen patients had FM/ CVT as treatment supporters with 6 (3.8\%), 93 (60\%), and 15 $(9.7 \%)$, respectively in the three age groups. Similarly, among the patients with "no treatment support" 40 were declared as treatment success, with the majority $30(63.8 \%)$ in the productive age group, while the remaining $10(21.3 \%)$ were in the older age group. It was noticed that interestingly all patients with age $<14$ years were provided with a treatment supporter and all were declared as treatment success. Among the patients with "unfavorable" and "transferred out" outcomes, the majority were belonged to the productive age group (Table-VII).

\section{Table-II}

Distribution of TB patients by geography with treatment supporter group.

\begin{tabular}{|c|c|c|c|c|}
\hline \multirow[t]{5}{*}{ Geography } & \multicolumn{4}{|c|}{ Treatment Supporter } \\
\hline & \multicolumn{3}{|c|}{ Treatment Support } & \multirow{3}{*}{$\begin{array}{c}\text { No Treatment Support } \\
\text { (self support }+ \text { undocumented) } \\
(47)\end{array}$} \\
\hline & LHW & CHW/HFW & $\mathrm{FM} / \mathrm{CVT}$ & \\
\hline & (203) & (46) & (155) & \\
\hline & $\mathrm{n}(\%)$ & $\mathrm{n}(\%)$ & $\mathrm{n}(\%)$ & $\mathrm{n}(\%)$ \\
\hline Urban & $74(36.5)$ & $21(45.7)$ & $112(72.3)$ & $34(72.3)$ \\
\hline Rural & $129(63.5)$ & $25(54.3)$ & $43(27.7)$ & $13(27.7)$ \\
\hline
\end{tabular}


Table-III

Distribution of TB patients by gender with treatment supporter group.

\begin{tabular}{lcccc}
\hline Gender & \multicolumn{3}{c}{ Treatment Supporter } \\
\cline { 2 - 5 } & \multicolumn{3}{c}{ Treatment Support } & No Treatment Support \\
\cline { 2 - 5 } & LHW & CHW/HFW & FM/CVT & (self support + undocumented) \\
& $(203)$ & $(46)$ & $(155)$ & $(47)$ \\
Male & $\mathrm{n}(\%)$ & $\mathrm{n}(\%)$ & $81(52.3)$ & $\mathrm{n}(\%)$ \\
Female & $102(50.2)$ & $25(54.3)$ & $74(47.7)$ & $29(61.7)$ \\
\hline
\end{tabular}

Table-IV

Distribution of TB patients by age group with treatment supporter group.

\begin{tabular}{lcccc}
\hline Age groups & \multicolumn{3}{c}{ Treatment Supporter } \\
\cline { 2 - 4 } & \multicolumn{3}{c}{ Treatment Support } & No Treatment Support \\
\cline { 2 - 5 } & LHW & CHW/HFW & FM/CVT & (self support + undocumented) \\
& $(203)$ & $(46)$ & $(155)$ & $(47)$ \\
\hline$<14$ yrs & $\mathrm{n}(\%)$ & $\mathrm{n}(\%)$ & $6(3.9)$ & $\mathrm{n}(\%)$ \\
$15-54$ yrs & $6(2.9)$ & $2(4.4)$ & $118(76.1)$ & $0(0)$ \\
$>55$ yrs & $137(67.5)$ & $34(73.9)$ & $31(20)$ & $35(74.5)$ \\
\hline
\end{tabular}

Table-V

Treatment outcomes by geography and treatment supporter distribution.

\begin{tabular}{llcccc}
\hline Outcomes & Geography & \multicolumn{3}{c}{ Treatment Supporter } \\
\cline { 3 - 6 } & & \multicolumn{3}{c}{ Treatment Support } & No Treatment Support \\
\cline { 3 - 6 } & & LHW & CHW/HFW & FM/CVT & (self support+ undocumented) \\
& & $(203)$ & $(46)$ & $(155)$ & $(47)$ \\
Treatment Success & Urban & $72(35.5)$ & $19(41.3)$ & $75(48.4)$ & $30(63.8)$ \\
& Rural & $117(57.6)$ & $22(47.8)$ & $39(25.1)$ & $10(21.3)$ \\
Unfavorable $\dagger$ & Urban & $1(0.5)$ & $1(2.2)$ & $20(13)$ & $3(6.4)$ \\
Transferred out & Rural & $9(4.4)$ & $1(2.2)$ & $1(0.6)$ & $0(0)$ \\
& Urban & $1(0.5)$ & $1(2.2)$ & $17(11)$ & $1(2.1)$ \\
\hline
\end{tabular}

$\dagger$ Unfavorable: It includes failure, defaulted and died cases.

Table-VI

Treatment outcomes by gender and treatment supporter distribution.

\begin{tabular}{llcccc}
\hline Outcomes & Gender & \multicolumn{3}{c}{ Treatment Supporter } \\
\cline { 3 - 5 } & & \multicolumn{3}{c}{ Treatment Support } & No Treatment Support \\
\cline { 3 - 5 } & & LHW & CHW/HFW & FM/CVT & (self support+undocumented) \\
& & $(203)$ & $(46)$ & $(155)$ & $(47)$ \\
Treatment Success & Male & $91(44.8)$ & $23(50)$ & $61(39.3)$ & $23(48.9)$ \\
Unfavorable & Female & $98(48.3)$ & $18(39.1)$ & $53(34.2)$ & $17(36.2)$ \\
Transferred out & Male & $7(3.4)$ & $1(2.2)$ & $14(9)$ & $3(6.4)$ \\
& Female & $3(1.5)$ & $1(2.2)$ & $7(4.6)$ & $0(0)$ \\
& Male & $4(2)$ & $1(2.2)$ & $6(3.8)$ & $3(6.4)$ \\
& Female & $0(0)$ & $2(4.3)$ & $14(9)$ & $1(2.1)$ \\
\hline
\end{tabular}


Table-VII

Treatment outcomes by age group and treatment supporter distribution.

\begin{tabular}{|c|c|c|c|c|c|}
\hline \multirow[t]{5}{*}{ Outcomes } & & \multicolumn{4}{|c|}{ Age groups Treatment Supporter } \\
\hline & & \multicolumn{3}{|c|}{ Treatment Support } & \multirow{4}{*}{$\begin{array}{c}\text { No Treatment Support } \\
\text { (self support+ undocumented) } \\
(47) \\
\mathrm{n}(\%)\end{array}$} \\
\hline & & LHW & CHW/HFW & $\mathrm{FM} / \mathrm{CVT}$ & \\
\hline & & (203) & (46) & $(155)$ & \\
\hline & & $\mathrm{n}(\%)$ & $\mathrm{n}(\%)$ & $\mathrm{n}(\%)$ & \\
\hline \multirow[t]{3}{*}{ Treatment Success } & $<14 \mathrm{yrs}$ & $6(3)$ & $2(4.3)$ & $6(3.8)$ & $0(0)$ \\
\hline & $15-54 \mathrm{yrs}$ & $130(64)$ & $29(63.1)$ & $93(60)$ & $30(63.8)$ \\
\hline & $>55 \mathrm{yrs}$ & $53(26.1)$ & $10(21.7)$ & $15(9.7)$ & $10(21.3)$ \\
\hline \multirow{3}{*}{ Unfavorable } & $<14$ yrs & $0(0)$ & $0(0)$ & $0(0)$ & $0(0)$ \\
\hline & $15-54 \mathrm{yrs}$ & $4(2)$ & $2(4.3)$ & $10(6.5)$ & $2(4.3)$ \\
\hline & $>55 \mathrm{yrs}$ & $6(2.9)$ & $0(0)$ & $11(7.1)$ & $1(2.1)$ \\
\hline \multirow[t]{3}{*}{ Transferred out } & $<14$ yrs & $0(0)$ & $0(0)$ & $0(0)$ & $0(0)$ \\
\hline & $15-54 \mathrm{yrs}$ & $3(1.5)$ & $3(6.6)$ & $15(9.7)$ & $3(6.4)$ \\
\hline & $>55 \mathrm{yrs}$ & $1(0.5)$ & $0(0)$ & $5(3.2)$ & $1(2.1)$ \\
\hline
\end{tabular}

\section{Discussions}

The national TB control program is not in the early stage in the country. The national program achieved 100\% TB DOTS country wide coverage in all public sector health facilities in 2005. The TB DOTS program was implemented in the district Rawalpindi in 2003.

\section{Treatment Support:}

Our study showed that the most preferred treatment supporters were lady health workers (45\%), followed by family members and community volunteers $(34.4 \%)$ and community health worker and health facility worker $(10.2 \%)$.

The common practice in NTP Pakistan for selection of suitable treatment supporter is that the DOTS facilitator conduct a dialogue with the TB patient and tries to convince $\mathrm{him} / \mathrm{her}$ to accept LHW as a treatment supporter. If the LHW is accessible and acceptable to the patient then she is requested to be the treatment supporter. But if the LHW is not available or not acceptable by the patient, then the patient is provided with another choice of $\mathrm{CHW} / \mathrm{HFW}$, and still if $\mathrm{CHW} / \mathrm{HFW}$ is not accessible or not acceptable by the patient then a FM/CVT is selected as a treatment supporter for the patient.

In our study we observed that the treatment supporter selection process was according to the national guidelines, but there were certain important issues which need program attention to streamline the operations related to the direct observation. These include: better mapping of the treatment supporters in the catchment area of the health facility and to update the lists of the treatment supporters periodically. In addition, training of different types of treatment supporters including LHW, CVT, and CHW.

The most important finding of our study was that a significantly higher number of rural patients preferred LHW as compared to the urban patients who preferred FM/CVT as their treatment supporters. This could be due to the social unacceptability to the patients or families and availability or non-availability of the LHW in the urban settings. Another important finding of the study was the higher numbers 324 (72.8\%) of cases from the productive age group (15-54 yrs). We also observed that the patients within the age group d" 14 yrs, all were provided with a treatment supporter.

\section{Treatment outcomes:}

Several randomized controlled trials have been done globally to look at the impact of treatment supporters on treatment outcomes. In our study, the overall treatment success rate was $85.1 \%$. This seemed to be according to the WHO target of $85 \%$. If we talk about the treatment success rate in patients with "treatment support" or "no treatment support", there was no difference as both had $85.1 \%$ treatment success rate. The treatment success rate in the LHW, CHW/HFW and FM/CVT was $93.1 \%, 89.1 \%$ and $73.5 \%$, respectively. Similar findings have been documented in a study carried out in Southern Thailand, 411 patients were enrolled in the study and $379(92 \%)$ were initially provided with DOT, while 32 $(8 \%)$ refused DOT (8). The overall treatment success was $85 \%$. In a randomized control trial in Pakistan, 497 adults, new smear positive TB patients were enrolled (9). 170 were 
assigned to health workers, 165 to family members and 162 were self-administered. The outcomes were found almost similar, with treatment success rates $67 \%, 62 \%$, and $65 \%$, respectively. This study showed that none of the three strategies was superior to the other. In our study, although the treatment success rate was the same $(85.1 \%)$ in patient with "treatment support" and "no treatment support", it was higher than in the randomized controlled trial in Pakistan.

A randomized control trial was conducted in Tanzania, in urban setting, to evaluate the effectiveness of community based DOT by using guardian and former TB patients compared with the hospital based DOT (10). A total of 587 new TB patients including pulmonary smear positive (322), pulmonary smear negative (182) and extrapulmonary (83) were enrolled. The treatment success rate among patients under community and health facility based DOT were $85 \%$ and $83 \%$, respectively. The findings suggested that both DOT strategies were equally effective for good treatment outcomes.

In a cluster-randomized control trial in ten hill and mountain districts of Nepal, 907 new sputum smear positive patients were recruited (11). 549 (61\%) were enrolled into community DOTS in five districts and 358 (39\%) were enrolled into family member DOTS group in five districts. The treatment success rate was $85 \%$ and $89 \%$ in community and family member based DOTS, respectively. Other studies in Malawi, Thailand, Senegal and South-eastern Brazil have also shown that family members as supervisors can play a useful role in DOT in the community $^{12-15}$.

A recent study in two rural districts of Southern Ethiopia included 318 smear positive cases ${ }^{16}$. The objectives of the trial were to see whether involvement of health extension workers could improve the case detection and treatment success rates. Health extension workers were trained for two days in the intervention group to focus on symptoms and transmission of TB, how to identify suspects, collect, and label, store and transport sputum specimens, administer DOT and follow patients during the treatment. Health extension workers in the control group were not trained like in the intervention. The treatment success rate was higher in the intervention than in the control group (89.3\% vs. 83.1\%). In our study the treatment success rate was significantly higher in patients supervised by LHW (93.1\%) type of treatment support followed by the CHW/HFW (89.1\%) and FM/CVT (73.5\%). While in patients with "no treatment support" treatment success rate was $85.1 \%$. But over all treatment success rate was $85.1 \%$.

In our study the treatment success rate was higher in rural than in urban patients ( $89.5 \%$ vs. $81.3 \%)$. This could be due to the social unacceptability to patients or their families and non-availability of LHW, CHW/HFW in the urban settings. These findings are quite different from the study carried out in three districts in Burkina Faso where geographical distance was seen to be a particular challenge for rural patients due to the lack of transport (17). The time and cost is another challenge for poor patients. ${ }^{18,19}$ It is observed that daily wagers prefer to seek health care after completing the day's work, by which time all the government health out-patient departments are closed.

In our study the treatment success rate was slightly higher in females than in males ( $86.9 \%$ vs. $83.5 \%)$. Similar findings have been documented in studies in Southern Thailand, Pakistan and Southern Ethiopia. ${ }^{8,9,16}$

\section{Conclusions:}

1. Most of the patients $(89.6 \%)$ were provided with treatment support with LHW (45\%), followed by FM/ CVT (34.4\%), CHW/HFW (10.2\%).

2. The priorities for type of treatment support are different in urban and rural settings. FM/CVT were found to be the main type of treatment support in urban, while LHW in the rural settings.

3. The treatment success rate was significantly higher in patients supervised by LHW (93.1\%) type of treatment support followed by the CHW/HFW (89.1\%) and FM/ CVT (73.5\%). While in patients with "no treatment support" treatment success rate was $85.1 \%$. But over all treatment success rate was $85.1 \%$.

\section{Authors' contributions:}

MHS has participated in study design, data entry and analysis, data interpretation, draft writing of the manuscript, and editing. OM has participated in study design, data analysis, data interpretation and editing whereas EQ and MAK have participated in data interpretation and editing. All authors read and approved the final manuscript.

\section{Ethical considerations:}

The protocol was approved by the National Bioethical Committee (NBC) Pakistan,

(Ref: No. 4-87/10/NBC-50/RDC/2429).

\section{Acknowledgments and funding:}

Our deepest appreciations to the National TB Control Program Pakistan for their support and guidance during the field work. This work was supported by grants from Norwegian State Educational Loan Fund (Lanekassen), Norway.

\section{Conflict of Interest : None}




\section{References}

1. World Health Organization. Global Tuberculosis Control: WHO report 2010, WHO/HTM/TB/2010. 7.

2. Parry $\mathrm{C}$ and Davies PD. The resurgence of tuberculosis. Soc Appl Bacteriol Symp Ser, 25: 23S-26S.

3. World Health Organization. Toman's tuberculosis case detection, treatment, and monitoring: questions and answers. WHO/HTM/TB/2004. 334.

4. Muncz M, Bergestrom K. A guide for tuberculosis treatment supporter, Stop TB WHO Geneva, Switzerland, WHO/CDS/ TB/2002. 300 .

5. World Health Organization. Treatment of tuberculosis: guidelines- $4^{\text {th }}$ ed. WHO/HTM/TB/2009. 420.

6. National TB Control Programme. Ministry of Health, Government of Pakistan, Refresher module for doctors. Nov. 2008.

7. Palomino JC, Leao SC, and Ritacco V. Tuberculosis 2007 from basic science to patient care. $1^{\text {st }}$ ed. 2007.

8. Pungrassami P, Johnson SP, Chongsuvivatwong V, Olsen J. Has directly observed treatment improved outcomes for patients with tuberculosis in Southern Thailand? Tropical Medicine and International Health 2002; 7 (3): 271-279.

9. Walley JD, Khan MA, Newell JN, Khan MH. Effectiveness of the direct observation component of DOTS for tuberculosis: a randomised controlled trial in Pakistan. The Lancet 2001; 357: 664-669.

10. Wandwalo E, Kapalata N, Egwaga S, Morkve O. Effectiveness of community-based directly observed treatment for tuberculosis in an urban setting in Tanzania: a randomised controlled trial, Int J Tuberc Lung Dis 2004; 8 (10): 1248-1254.

11. Newell JN, Baral SC, Pande SB, Bam DS, Malla P. Familymember DOTS and community DOTS for tuberculosis control in Nepal: cluster-randomised controlled trial. The Lancet 2006; 367 (9514): 903-909.

12. Manders AJE, Banerjee A, Van Den Borne HW, Harries AD, Kok GJ, Salaniponi FML. Can guardians supervise TB treatment as well as health workers? A study on adherence during the intensive phase. Int J Tuberc Lung Dis 2001; 5 (9): 838-842.

13. Akksilp S, Rasmithat S, Maher D, Sawert H. Direct observation of tuberculosis treatment by supervised family members in Yasothorn Province, Thailand. Int J Tuberc Lung Dis 1999; 3 (12): 1061-1065.

14. Thiam S, LeFevre AM, Hane F, Ndiaye A, Ba F, Fielding $\mathrm{KL}$, et al. Effectiveness of a strategy to improve adherence to tuberculosis treatment in a resource-poor setting: A cluster randomized controlled trial. JAMA 2007; 297 (4): 380-386.

15. Maciel ELN, Guidoni LM, Brioshi AP, Prado TND, Fregona G, Hadad DM, et al. Household members and health care workers as supervisors of tuberculosis treatment. Rev Saude Publica 2010; 44 (2): 339-343.

16. Datiko DG, Lindtjørn B. Health extension workers improve tuberculosis case detection and treatment success in Southern Ethiopia: a community randomized trial. PLoS ONE 2009; $4: 5$.

17. Sanou A, Dembele M, Theobald S, Macq J. Access and adhering to tuberculosis treatment: barriers faced by patients and communities in Burkina Faso. Int J Tuberc Lung Dis 2004; 8 (12): 1479-1483.

18. Khan MA, Walley JD, Witter SN, Imran A, Safdar N. Cost and cost-effectiveness of different DOT strategies for the treatment of tuberculosis in Pakistan. Health Policy and Planning 2002; 17 (2): 178-186.

19. Xu Weiguo, Lu Wei, Zhou Y, Zhu L, Shen H, Wang J. Adherence to anti-tuberculosis treatment among pulmonary tuberculosis patients: a qualitative and quantitative study. BMC Health Services Research 2009; 9 : 169 